Int. J. Dev. Biol. 60: 221-227 (2016)

doi: $10.1387 / \mathrm{ijdb} .160103 \mathrm{vc}$

\title{
Studying essential DNA metabolism proteins in Xenopus egg extract
}

\author{
VINCENZO SANNINO, ARUN M. KOLINJIVADI, GIORGIO BALDI and VINCENZO COSTANZO* \\ DNA metabolism Laboratory, IFOM, The FIRC institute of Molecular Oncology, Milan, Italy
}

\begin{abstract}
The correct duplication of genetic information is essential to maintain genome stability, which is lost in cancer cells. Replication fork integrity is ensured by a number of DNA metabolism proteins that assist replication of chromatin regions difficult to replicate due to their intrinsic DNA sequence composition, coordinate repair of DNA molecules resulting from aberrant replication events or protect replication forks in the presence of lesions impairing their progression. Some DNA metabolism genes involved in DNA repair are essential in higher eukaryotes even in unchallenged conditions, suggesting the existence of biological processes requiring these specialized functions in organisms with complex genomes. The impact on cell survival of null mutants of many DNA metabolism genes has precluded complete in depth analysis of their function. Cell free extracts represent a fundamental tool to overcome survival issues. The Xenopus laevis egg cell free extract is an ideal system to study replication-associated functions of essential genes. We are taking advantage of this system together with innovative imaging and proteomic based experimental approaches to characterize the molecular function of essential DNA metabolism proteins. Using this approach we have uncovered the role of some essential homologous recombination and fork protection proteins in chromosomal DNA replication and we have characterized some of the factors required for faithful replication of specific vertebrate genomic regions. This approach will be instrumental to study the molecular mechanisms underlying the function of a number of essential DNA metabolism proteins involved in the maintenance of genome stability in complex genomes.
\end{abstract}

KEY WORDS: Xenopus laevis, immuno-depletion, DNA replication, DNA recombination and reversed forks

\section{Introduction}

Genome duplication is a key event in the life cycle of all biological organisms and its careful control is essential to preserve the physical integrity of chromosomes. DNA replication is carried out by the replisome machinery, which assembles at replication origins, where the Origin Recognition Complex (ORC) transiently associates with the Cdc6 initiator, which together with Cdt1 recruits the MCM2-7 complex acting as DNA helicase. Additional essential cofactors such as Treslin, RecQ4L and others are required for origin firing. Additional factors travel with the Replisome at the fork. Helicase activation and DNA unwinding depends on the S-phase kinase dependent association of the replisome component, Cdc45, with MCM2-7 and the concomitant recruitment of the GINS proteins, together forming the CMG complex. DNA unwinding is followed by Pol $\varepsilon$ and Pol $\alpha /$ Primase loading onto replication forks (Diffley, Diffley, 2004). The Replication Pausing Complex, composed of Tipin, Tim, And1 and Claspin proteins play a primary role in maintaining chromosomal integrity under replication stress conditions, as they keep the CMG from translocating, when replicative polymerases stall (Errico and Costanzo, 2012).

Maintenance of genome integrity during DNA replication relies on several factors such as the ability to respond to DNA lesions impairing fork progression, the coordination of DNA repair and DNA replication and the availability of DNA replication origins. These mechanisms are believed to be particularly important for the replication of specific chromosome regions with complex or repetitive DNA sequence composition, which are often involved in mutational events responsible for several human diseases (Lana et al., Ozeri-Galai et al.).

Establishment of replication origins in eukaryotes is regulated

\footnotetext{
Abbreviations used in this paper: EM, electron microscopy; HR, homologous recombination; MCM, mini chromosome mantainance; ORC, origin recognition complex; Pol, polymerase; ssDNA, single stranded DNA.
}

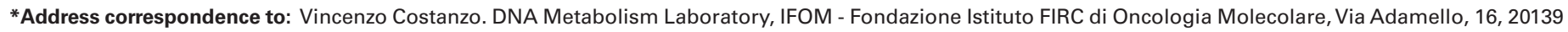
Milano MI, Italy. Tel. +39-02-574-303-875. Fax +39-02-574-303-231. E-mail:Vincenzo.costanzo@ifom.eu
}

Accepted: 14 April 2016.

ISSN: Online 1696-3547, Print 0214-6282 
by DNA sequences with differential affinities for ORC, chromatin structure, DNA methylation status, histone modifications and nuclear organization (Bell and Dutta, 2002). However, except for some eukaryotic model systems among which $S$. cerevisiae, where a specific DNA sequence dictates the specificity of ORC binding to DNA (Diffley, Diffley, 2004) the mechanism of replication origin placement and distribution in animal cell genome is unclear. In addition, many replication origins remain "dormant" and are activated only in the presence of DNA lesions or structures that perturb replication fork progression to ensure complete replication of the affected DNA segment (Woodward et al., 2006).

The mechanisms underlying the function of DNA repair and DNA damage response proteins in unchallenged chromosomal DNA replication are poorly understood. This is in part due to the fact that many of the genes involved in DNA metabolism are essential for life, especially in higher eukaryotes.

Cell free systems based on vertebrate Xenopus laevis egg extract have been helpful to elucidate the biochemical bases of cell cycle checkpoints, DNA repair and DNA replication (Balestrini et al., Costanzo et al., 2001, Costanzo et al., 2004, Costanzo et al., 2000, Errico et al., 2009, Errico et al., 2007, Garner and Costanzo, 2009, Hashimoto et al., Hashimoto and Costanzo, Hashimoto et al., Lazzaro et al., Shechter et al., 2004, Trenz et al., 2008, Trenz et al., 2006). This system can be defined as "liquid life" or "life in a tube" as it can reproduce basic events in the cell life such as cell cycle, chromatin formation, nuclear assembly, semi-conservative DNA replication, chromosome condensation and mitotic spindle assembly driven by cyclin synthesis and degradation cycles. The high degree of genetic conservation between Xenopus and mammalian organisms facilitates the study of large proteins present in egg extracts. We have successfully combined the use of the Xenopus egg extract with advanced imaging techniques based on transmission electron microscopy (EM) to visualize replication intermediates obtained from genomic DNA replicated in extracts depleted of particular proteins. This approach has uncovered a role for Rad51 in preventing the occurrence of single stranded DNA (ssDNA) gaps during replication and for PARP1 in controlling the formation of reversed replication forks, which are DNA replication intermediates whose processing is critical for genome stability maintenance (Hashimoto et al., Ray Chaudhuri et al.). Furthermore, we established a primary role for Rad51 and the Mre11-Rad50-Nbs1 complex restarting replication forks collapsed by a nick in the DNA template (Hashimoto et al.). Here we will review the recent work and propose new research avenues which could take advantage of cell free extracts.

\section{Basic tools to study protein function with Xenopus egg extract}

Xenopus egg extract is capable of recapitulating many aspects of cell biology due to the exceptional richness of proteins and RNA molecules stored as maternal stockpile. In the embryo these molecules are gradually made available during rapid cell cycles, which proceed in the absence of any significant level of transcription until mid-blastula transition. Due to these features complex phenomena such as DNA replication can take place in egg extract just by adding sperm nuclei and other DNA templates. The use and the production of different types of Xenopus egg extracts are based on established protocols (Costanzo and Gautier,
2004, Costanzo et al., 2004, Hashimoto and Costanzo, 2011). A major tool available to study protein function in cell free extracts is antibody-mediated protein depletion. Selective protein depletion from egg extract allows the characterization of the biochemical steps in which specific proteins operate. The application of this procedure to essential DNA metabolism proteins facilitates the study of vertebrate gene products that would normally compromise viability when inactivated in other cellular systems, especially during chromatin replication. Optimal protein depletion relies on high affinity antibodies. In our experience polyclonal rabbit antibodies produced against the whole or $\mathrm{N}$-terminus and $\mathrm{C}$-terminus exposed portions of Xenopus proteins work better than mouse monoclonal antibodies for depletion and are easier to obtain. However, high quality antibodies are sometime difficult to produce and require several trials to identify the antigen with the highest immunogenicity. Purification of specific immunoglobulins with the highest binding affinity for the target increases depletion power of the antibodies. Once good high affinity antibodies are generated depletion experiments are relatively straightforward to perform.

There are some important considerations to make on protein depletion. In cells inactivation of specific proteins relies on techniques such as gene knockout or knockdown, which operate before the protein is produced. This is clearly not possible in egg extracts in which proteins are already made. However, high affinity antibodies can usually deplete up to $95-100 \%$ of defined proteins, depending on the affinity of the antibodies and the extract concentration of the factor to be depleted. Proteins often operate in large multi-protein complexes. It is commonly assumed that antibodies directed against a member of the complex can deplete the whole complex (Fig 1). This is in part true with complexes that are extremely stable such the Mre11-Rad50 complex. Usually, this happens when proteins complexes can withstand high salt conditions in vitro. In our experience antibodies with high binding affinities can also single out proteins from protein complexes, selectively displacing and depleting individual subunits. This is achieved by overcoming the dynamic binding equilibrium among the different components of a single complex (Fig 1). It is relatively easy to monitor the status of the remaining components of the complex in the extract by western blot. This is an important point to consider when studying proteins acting in different complexes. As such the antibody-mediated depletion procedure can be as powerful as other techniques to inactivate single protein functions. Complementation of the depleted extract with recombinant proteins can be performed to validate the effect of the depletion. However, not always sufficient amounts of active and properly folded recombinant proteins are available for this control. In this case protein depletion can be validated with different sets of antibodies directed against the same factor to make sure that the phenotypic effects observed following protein depletion are due to the removal of the specific factor and not of other unrelated proteins. It is indeed highly unlikely that different sets of antibodies directed against the same factor will have similar off-targets. The rationale underlying this validation protocol is the same of gene knockdown procedures with different sets of oligonucleotides. The ability of egg extract to produce proteins from mRNA translation or even from transcription coupled translation of specific cDNA (Tokmakov et al., 2006) can also be used to complement depletion experiments, especially with factors whose extract concentration is compatible with the restoration achievable with these procedures. Alternatively, it is also possible to purify active protein complexes or proteins to 
homogeneity directly from Xenopus egg extract. Several rounds of chromatographic fractionation techniques can be employed to obtain relatively pure protein from extract. Purification of proteins from egg extract is often successful for highly abundant factors.

A major issue of rabbit polyclonal antibodies is their limiting amount, which hampers the possibility of sharing the tool in sufficient amounts to perform depletion experiments. This issue is not present with other genetically modifiable model systems such as worms, flies and yeast in which mutants can be easily distributed. Therefore, due to the unpredictable nature of antibodies quality and availability alternative approaches should be considered when studying protein function in cell free extracts. Furthermore, in some instances due to high endogenous concentration (e.g. histones) complete protein depletion is not even achievable. In these cases it is possible to overstuff the extract with recombinant wild type or mutant proteins, which displace the endogenous proteins in the molecular steps and complexes in which they are involved (Fig 1). This is especially useful when proteins perform their function on chromatin. In these cases the recombinant protein can entirely substitute the endogenous one on chromatin. We have previously used this approach to study the effect of mutant PCNA protein subunits on chromatin. As redundant post replication repair pathways such as translesion synthesis mask the role of homologous recombination in replication fork progression, we adopted this approach to inhibit translesion synthesis using a recombinant mutant PCNA (PCNA K164R) that cannot be ubiquitinated and therefore does not fully support binding of translesion polymerase Pol $\eta$ to chromatin (Hashimoto et al., 2010). More recently, we have used a similar strategy to study different Cdc45 protein domains, whose function had been predicted based on Cdc45 arrangement in the context of the CMG complex.
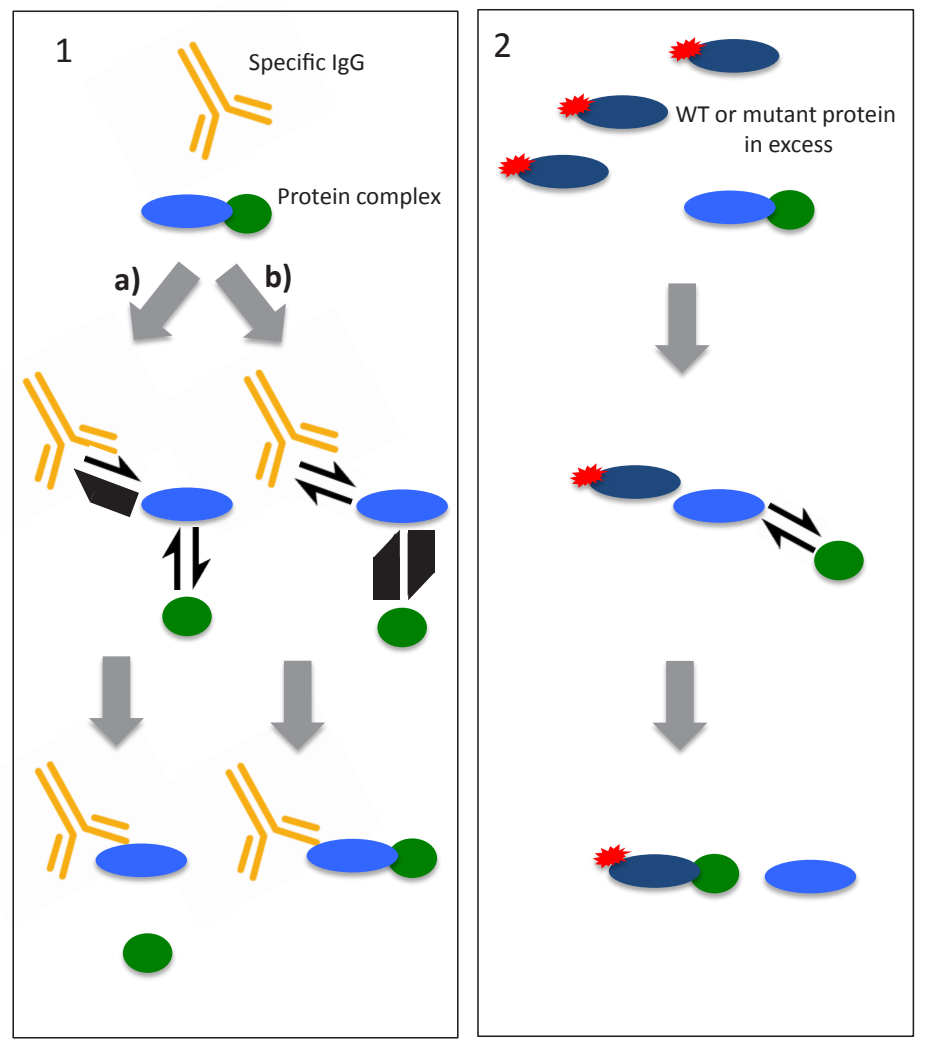

Interference on protein function can also be obtained with small peptides that bind at the interface of the protein-protein interaction site like we did to study the replication function of Rad51. It this study, we successfully inhibited Rad51 binding to chromatin using recombinant human BRC4 (one of eight BRC motifs of BRCA2 that has a strong affinity for Rad51) (Hashimoto et al., 2010). We demonstrated that BRC4 or Rad51 depletion had similar effects on DNA replication, further validating this approach.

\section{Understanding the role of essential DNA recombina- tion and fork protection proteins in chromosomal DNA replication}

HR genes such as BRCA1, BRCA2 and the Rad51 paralog family, which consists in mammalian cells of five proteins (Rad51B, Rad51C, Rad51D, Xrcc2 and Xrcc3), are required to repair ionizing radiation-induced DNA damage by homologous recombination. Mutations in these genes predispose to cancer, indicating an important role in preventing or repairing DNA damage predisposing to cell transformation. Intriguingly, complete loss of function of most of HR proteins is incompatible with life in vertebrate organisms or leads to severe chromosome breakage, cell cycle arrest and apoptosis in cellular model systems. For example, deletion (Errico and Costanzo, 2012) or functional inactivation (Buis et al., 2008) of homologous recombination (HR) genes such as Rad51, Mre11, Rad50 or Nbs 1 result in lethality in mice. In contrast, mutations of the orthologs of these genes in S. cerevisiae have limited effects and null cells are mostly viable in the absence of exogenous DNA damaging insults (D'Amours and Jackson, 2002). Therefore, while yeast mutants in many of the key DNA metabolism genes are viable, loss of the same proteins in higher eukaryotes results in cell

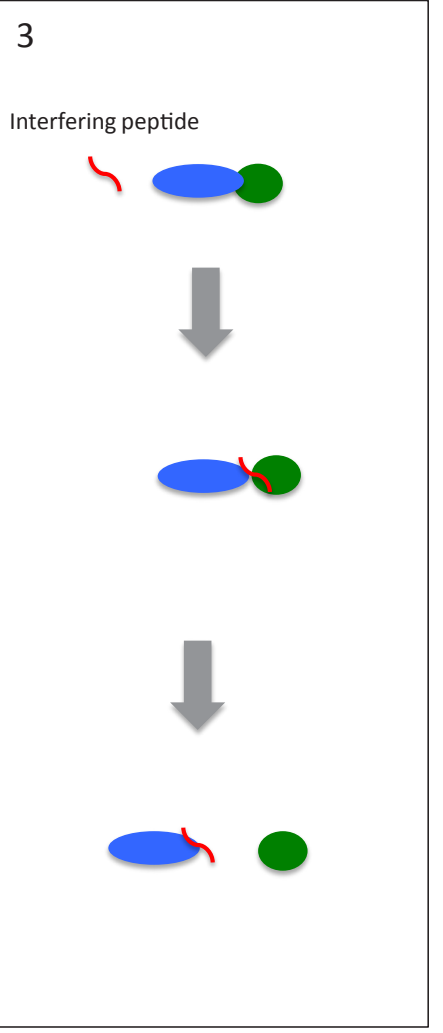

Fig. 1. Removing and inactivating proteins from cell free extracts. (1) Antibodies directed against single subunits of protein complex can single out and deplete individual components (a) or the whole complex (b) depending on the affinity and ability of the antibody to overcome binding equilibrium between complex components. (2) Recombinant mutant or WT proteins added to egg extract in excess could substitute individual protein subunits by protein exchange. (3) Peptide binding at the interphase of protein-protein interaction domains can disrupt interaction between protein complex subunits. 
or embryonic lethality. Furthermore, DNA repair is more complex in higher eukaryotes given the presence of additional essential genes such as BRCA2 and BRCA1, which regulate several aspects of $H R$ (Liu and West, 2002, Thorslund and West, 2007). The reason why these genes are essential for life in higher eukaryotes is unclear. One explanation might be that they have a specific role in the replication of complex genomic regions typical of higher eukaryotes. Alternatively, complex genomes might be more vulnerable to spontaneous damage that might irreversibly halt replication progression and therefore might require HR factors to complete DNA replication. This would be consistent with the observations that chromosomal aberrations are frequent in vertebrate cells with mutated HR proteins including BRCA2, BRCA1 and Rad51 paralogs. These features together with their ability to form foci in unperturbed S-Phase indicate a possible role for HR proteins in chromosomal replication even in unchallenged conditions (Aly and Ganesan, Costanzo, Jasin, 2002, Ohta et al., Suwaki et al., West, 2003). As these functions are essential for cell duplication and cell survival we thought that the use of cell free extracts and single molecule analysis could have been helpful to understand HR protein function in DNA replication. Therefore, to study the role of these DNA metabolism proteins in vertebrate DNA replication we set up biochemical assays based on Xenopus egg extract coupled to the analysis of replication intermediates with single molecule imaging techniques. These procedures allow visualization of the native DNA configuration of replication intermediates isolated from egg extract from which specific factors have been depleted. To this end DNA are subjected to in vivo crosslinking, obtained by exposing sperm nuclei replicated in egg extract to trimethylpsoralen (TMP), followed by irradiation pulses with $365-366 \mathrm{~nm}$ monochromatic light. This treatment induces the formation of interstrand crosslinks, preventing branch migration of DNA and therefore stabilizing replication intermediates. DNAisolated from egg extract is then deproteinized, enriched on DNA binding columns and layered onto EM grids, which are then processed by rotary shadowing to visualize the DNA molecules (Lopes, 2009, Ray Chaudhuri et al.). This type of analysis can be easily performed in Xenopus egg extract and it is facilitated by the smaller size of the replicon compared to somatic cells and by the abundance of replication intermediates.

\section{The ability to image DNA replication intermediates and to easily perturb the system by adding and depleting proteins or DNA damaging agents provides single molecule level information on the function of DNA me- tabolism proteins}

Using EM based analysis of replication intermediates we found that Rad51 prevents the accumulation of ssDNA gaps at replication forks and behind them during DNA replication (Hashimoto et al.). ssDNA gaps behind forks can be suppressed by the inhibition of Mre11 activity indicating a role for Mre11 in mediating the formation of these structures. Our findings were independently confirmed in cells defective for Rad51 DNA loading factor BRCA2 (Jensen et al., Thorslund et al.), which show Mre11 dependent degradation of stalled replication forks (Schlacher et al.). It is possible that BRCA2 or Rad51 paralogs load Rad51 onto gaps that arise behind replication forks during unchallenged or perturbed DNA replication. The accumulation of ssDNA gaps might reflect the discontinuous
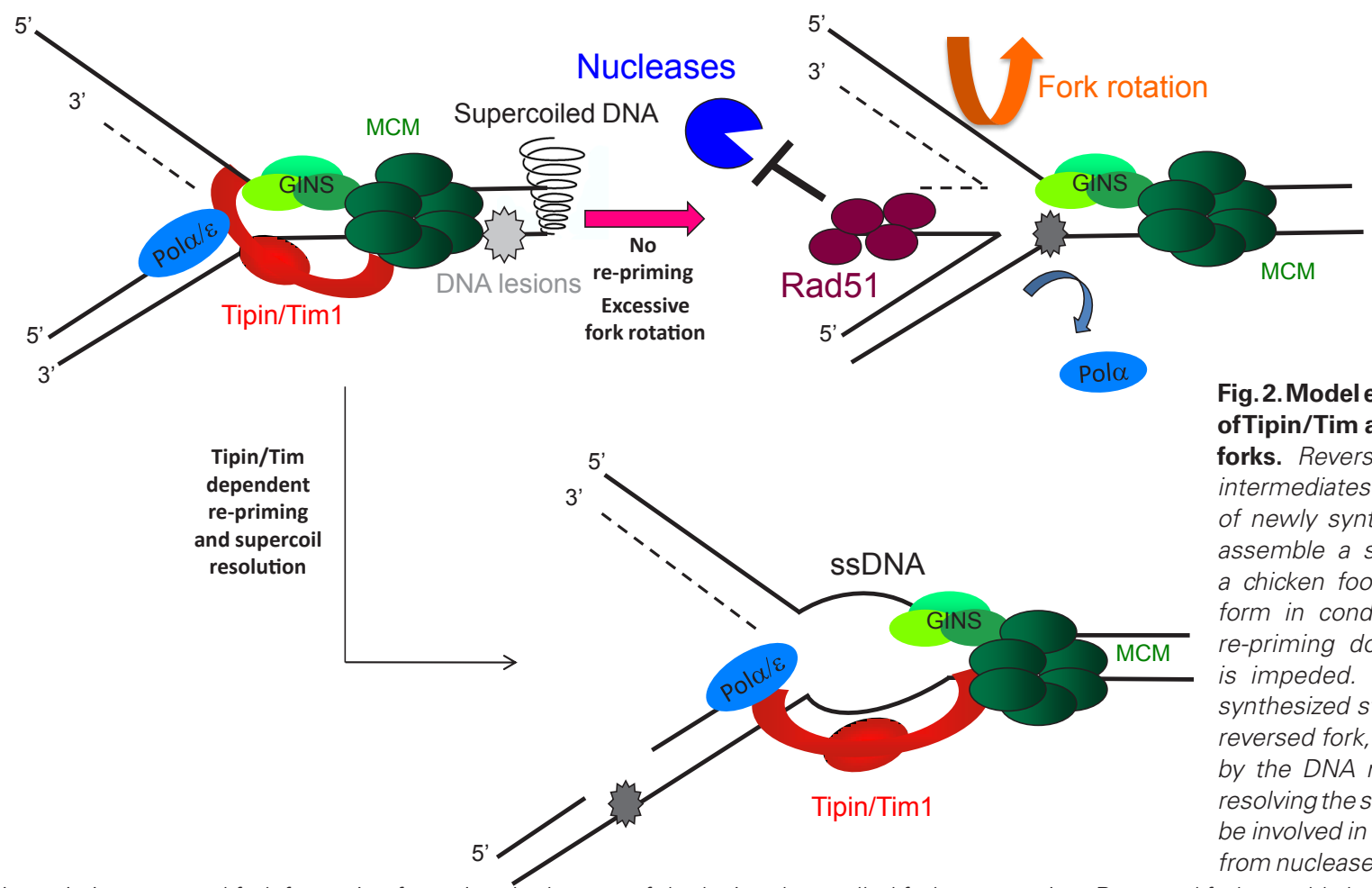

Fig. 2. Model explaining possible roles ofTipin/Tim and Rad51 at replication forks. Reversed forks are replication intermediates formed by the annealing of newly synthesized DNA strands to assemble a structure that resembles a chicken foot. Reversed forks might form in conditions where replication re-priming downstream fork stalling is impeded. In this case the newly synthesized strands anneal and form a reversed fork, which can be processed by the DNA repair machinery to help resolving the stalling event. Rad51 might be involved in protecting reversed forks from nuclease-mediated degradation or

in assisting reversed fork formation favouring the bypass of the lesion that stalled fork progression. Reversed forks could also form to equilibrate unresolved DNA supercoiling accumulating ahead of forks in the absence of functional Tipin/Tim complex, which might promote resolution of the positive supercoiling by relocating Topoisomerases to replication intermediates (See text for additional explanation). In the absence of Tipin/Tim spontaneous reversed forks accumulation and/or fork rotation would dissipate positive supercoiling behind forks, facilitating the release of replisome components. 
nature of replication fork progression. Gaps might be formed by polymerase mediated re-priming in replication bubbles downstream the replication block to overcome fork arrest (Fig 2). Replisome components are usually stably associated to replication forks (Errico and Costanzo, 2010, Errico and Costanzo, 2012) and the Tipin/ Tim complex is required for efficient polymerase alpha binding to chromatin. We have shown that the Tipin/Tim complex promotes polymerase alpha dependent replication re-priming (Errico et al., 2014). Using EM we found that a large fraction of replication intermediates in Tipin/Tim depleted extracts contains reversed forks, which are replication intermediates formed by the annealing of newly synthesized DNA strands to assemble a structure that resembles a chicken foot (Errico et al., 2014) (Fig 2). This structure might form when replication re-priming downstream fork stalling is impeded. In this case the newly synthesized strands anneal and form a reversed fork, which can be processed by the DNA repair machinery to help resolving the stalling event. Rad51 might be involved in protecting reversed forks from nuclease-mediated degradation or in assisting reversed fork formation favouring the bypass of the lesion that stalled fork progression (Fig 2).

A role in preventing excessive fork rotation in the presence of replication fork roadblocks has been proposed for the Tipin/Tim complex in yeast (Schalbetter et al., 2015) (Fig 2). This function could be particularly important when Topoisomerases cannot remove positively supercoiled DNA accumulating ahead of replication forks, which prevents their progression if not resolved. Tipin/Tim dependent relocalization at forks of Topoisomerases, which have high affinity for the complex, might resolve the positive supercoiled DNA accumulation allowing fork progression. In the absence of Tipin/Tim reversed forks or precatenanes, which form following fork rotation, might accumulate behind forks, equilibrating the positive supercoiling present ahead of forks. This might lead to the release of Polymerase alpha, whose binding is destabilised in these conditions, as we previously showed. It is worth mentioning that continuous priming activity is a pre-requisite for checkpoint activation during fork stalling conditions. Loss of Tipin/Tim leads to severe impairment of Chk1 phosphorylation compatibly with the function of this complex in promoting primase activity at forks (Errico and Costanzo, 2010, Errico and Costanzo, 2012).

Reversed forks in Tipin/Tim depleted extracts have been observed in unchallenged conditions. However, Xenopus egg extract can be easily manipulated to study the effects of different DNA damaging agents on the frequency and the morphology of these structures. This approach might yield more insights on the pathways and the DNA structures leading to reverse fork formation. Importantly, qualitative analysis of replication intermediates can be easily complemented by statistical quantification of the different DNA structures observed with EM by scoring several fork structures per sample. This is facilitated by the abundance of intact replication intermediates that can be obtained from Xenopus egg extract (Hashimoto et al.), in which the frequency of replication origins is ten fold higher than somatic cells.

Interestingly, DNA bound to nucleosome is not efficiently crosslinked by psoralen and this feature can be exploited to visualize nucleosome distribution on normal and damaged replication forks (Lopes, 2009). Under denaturing conditions, DNA strands are separated where a nucleosome was positioned in vivo and are kept together by the interstrand crosslinks induced in each linker DNA tract. This gives to double-stranded DNA the appearance of a string of single-stranded DNA bubbles (Lopes, 2009) that allows the detection of nucleosomal array organization in correspondence of replication forks. This protocol could be useful to monitor the effects of HR factors, fork protection proteins and chromatin remodelling genes on nucleosome positioning at replication forks and termination zones.

A large fraction of reversed forks that we observed in Tipin/Tim depleted extracts contain a reversed branch made of ssDNA (unpublished data), which can be formed by the activity of nucleases such as Mre11. Inhibition of Mre11 activity with Mirin (Dupre et al., 2008) or its depletion from egg extract with specific antibodies could highlight the molecular mechanism leading to the formation of these structures. Additional factors that might be involved in the stabilization of the reversed forks such as Non Homologus End Joining repair (NHEJ) Ku70/80 and the DNA-PK proteins, which have high affinity for DNA end of the reversed branch (Foster et al.). Their role could be easily tested with the Xenopus cell free system.

Overall, the approach that we described will gradually reveal fundamental new molecular links bewteen DNA damage response and repair proteins and the DNA replication machinery.

\section{Identification and characterization of factors required for faithful replication of specific chromosome loci}

It is possible the essential role of some DNA metabolism genes in higher eukaryotes is related to their requirement for faithful replication of complex chromosomal regions present in the species in which these genes are required for cell viability. Consistent with this hypothesis HR factors such as BRCA1 have been found on pericentric heterochromatin and major DNA satellite blocks during unperturbed DNA replication (Pageau and Lawrence, 2006). BRCA1 has also been shown to localized to recently identified to early fragile sites containing complex repeated sequences such as retrotransposons, tRNA genes, LINE and SINE elements (Barlow et al., 2013), which constitute natural impediments to replication fork progression, giving rise to chromosomal translocations in cancer cells (Mirkin and Mirkin, 2007). To identify and characterize proteins necessary for replication of specific genomic sequences and verify the role of HR factors in this process we have developed a system to support in vitro replication of defined DNA sequences in Xenopus egg extract using large DNA bacmids (size between 120-250 Kb) containing different human DNA genomic sequences (Errico et al., 2014). Bacmids can be used to probe the replication of specific DNA sequences associated to different chromatin regions such as centromeres, minisatellite DNA and known fragile sites containing variable numbers of complex or repeated DNA sequences. In contrast to small plasmids, which do not support efficient nucleus formation and semi-conservative DNA replication in egg cytoplasm and consistent with the fact that large size plasmids replicate more efficiently when injected into Xenopus eggs (Harland and Laskey, 1980, Mechali and Kearsey, 1984, Sanchez et al., 1992), we have shown that large bacmids support nucleus formation and origin-dependent DNA replication with high efficiency in egg extract with up to $70 \%$ of the input molecules fully replicated. Nuclei formed by bacmid DNA can be isolated at different time points following their addition to egg extract by sucrose gradient centrifugation and the status of DNA binding proteins required for the assembly of replication origin such as ORC1-6 or MCM2-7 and DNA replication initiation such as CDC45 and GINS can be 
monitored by western blot to identify the stage of DNA replication reaction in which they are involved (Errico and Costanzo, 2010). For these experiments human genomic DNA cloned in the human bacmid library can be used. Using this system secondary non-B DNA structures, which interfere with higher-order chromatin folding could be studied to understand how they affect the replication dynamics and nucleosome assembly, resulting in decondensation defects seen as fragile sites (Lukusa and Fryns, 2008). The same analysis could be extended to other complex human and Xenopus genomic DNA sequences containing repetitive elements. In preliminary experiments we have shown that human centromeric DNA is able to promote selective loading of centromeric protein CENP-A, a histone variant mainly found at centromeres. This result indicates that human centromeric DNA and possibly other human genomic DNA sequences are able to promote specific chromatin conformations and can be used as template to study replication of centromeric or other specific chromatin regions in Xenopus egg extract. This system could be used to test the hypothesis that HR and fork protection proteins are required for efficient and faithful replication of genomic regions containing repetitive sequences. Replicated bacmids could be subjected to PCR and southern blot based analysis to uncover possible abnormalities in copy numbers and sequence rearrangements following replication in the absence of HR and fork stabilizing factors. This analysis could be complemented by deep sequencing of replicated DNA to monitor fidelity of DNA synthesis. Overall, these experiments could help uncovering for the first time and on a large scale the potential role of known and novel DNA and chromatin metabolism genes in faithful replication of complex chromosomal regions.

\section{Final remarks}

Xenopus cell-free extract represents one of most powerful tools available to study replication of vertebrate genomes in unchallenged and stressful conditions. This system will be helpful to clarify the role of several essential DNA metabolism genes with a potential involvement in different aspects of DNA replication. Classical biochemical assays coupled to novel imaging techniques could lead to several groundbreaking discoveries, including a better understanding of genomic DNA and chromatin metabolism at specific genomic DNA loci. These integrated approaches will greatly contribute to understand molecular mechanisms underlying the function of essential DNA metabolism genes in the replication and repair of vertebrate genomes.

\section{Acknowledgements}

We thank members of the Costanzo lab for helpful discussions. Work described here is funded by the Associazione Italiana per Ricerca sul Cancro (AIRC), the European Research Council (ERC) Consolidator grant (614541), the Giovanni Armenise-Harvard foundation award, the Epigen Progetto Bandiera (4.7), the AICR-Worldwide Cancer Research (13-0026) and the Fondazione Telethon (GGP13-071) grant awarded to V.C.

\section{References}

ALY, A. and GANESAN, S. BRCA1, PARP, and 53BP1: conditional synthetic lethality and synthetic viability. J Mol Cell Biol 3: 66-74.

BALESTRINI, A., COSENTINO, C., ERRICO, A., GARNER, E. and COSTANZO, V. GEMC1 is a TopBP1-interacting protein required for chromosomal DNA replication. Nat Cell Biol 12: 484-491.

BARLOW, J.H., FARYABI, R.B., CALLEN, E., WONG, N., MALHOWSKI, A., CHEN,
H.T., GUTIERREZ-CRUZ, G., SUN, H.W., MCKINNON, P., WRIGHT, G. et al., (2013). Identification of Early Replicating Fragile Sites that Contribute to Genome Instability. Cell 152: 620-632

BELL, S.P. and DUTTA, A. (2002). DNA replication in eukaryotic cells. Annu Rev Biochem 71: 333-374

BUIS, J., WU, Y., DENG, Y., LEDDON, J., WESTFIELD, G., ECKERSDORFF, M., SEKIGUCHI, J.M., CHANG, S. and FERGUSON, D.O. (2008). Mre11 nuclease activity has essential roles in DNA repair and genomic stability distinct from ATM activation. Cell 135: 85-96.

COSTANZO, V. Brca2, Rad51 and Mre11: performing balancing acts on replication forks. DNA Repair (Amst) 10: 1060-1065

COSTANZO, V. and GAUTIER, J. (2004). Xenopus cell-free extracts to study DNA damage checkpoints. Methods Mol Biol 241: 255-267.

COSTANZO, V., ROBERTSON, K., BIBIKOVA, M., KIM, E., GRIECO, D., GOTTESMAN, M., CARROLL, D. and GAUTIER, J. (2001). Mre11 protein complex prevents double-strand break accumulation during chromosomal DNA replication. Mol Cell 8: 137-147.

COSTANZO, V., ROBERTSON, K. and GAUTIER, J. (2004). Xenopus cell-free extracts to study the DNA damage response. Methods Mol Biol 280: 213-227.

COSTANZO, V., ROBERTSON, K., YING, C.Y., KIM, E., AVVEDIMENTO, E., GOTTESMAN, M., GRIECO, D. and GAUTIER, J. (2000). Reconstitution of an ATMdependent checkpoint that inhibits chromosomal DNA replication following DNA damage. Mol Cell 6: 649-659.

D'AMOURS, D. and JACKSON, S.P. (2002). The Mre11 complex: at the crossroads of dna repair and checkpoint signalling. Nat Rev Mol Cell Biol 3: 317-327.

DIFFLEY, J.F. Quality control in the initiation of eukaryotic DNA replication. Philos Trans R Soc Lond B Biol Sci 366: 3545-3553.

DIFFLEY, J.F. (2004). Regulation of early events in chromosome replication. Curr Biol 14: R778-R786.

DUPRE, A., BOYER-CHATENET, L., SATTLER, R.M., MODI, A.P., LEE, J.H., NICOLETTE, M.L., KOPELOVICH, L., JASIN, M., BAER, R., PAULL, T.T. et al., (2008). A forward chemical genetic screen reveals an inhibitor of the Mre11-Rad50-Nbs1 complex. Nat Chem Biol 4: 119-125.

ERRICO, A., AZE, A. and COSTANZO, V. (2014). Mta2 promotes Tipin-dependent maintenance of replication fork integrity. Cell Cycle 13: 2120-2128.

ERRICO, A., COSENTINO, C., RIVERA, T., LOSADA, A., SCHWOB, E., HUNT, T. and COSTANZO, V. (2009). Tipin/Tim1/And1 protein complex promotes Po alpha chromatin binding and sister chromatid cohesion. EMBO J 28: 3681-3692.

ERRICO, A. and COSTANZO, V. (2010). Differences in the DNA replication of unicellular eukaryotes and metazoans: known unknowns. EMBO Rep 11: 270-278.

ERRICO, A. and COSTANZO, V. (2012). Mechanisms of replication fork protection: a safeguard for genome stability. Crit Rev Biochem Mol Biol 47: 222-235.

ERRICO, A., COSTANZO, V. and HUNT, T. (2007). Tipin is required for stalled replication forks to resume DNA replication after removal of aphidicolin in Xenopus egg extracts. Proc Natl Acad Sci USA 104: 14929-14934.

FOSTER, S.S., BALESTRINI, A. and PETRINI, J.H. Functional interplay of the Mre11 nuclease and $\mathrm{Ku}$ in the response to replication-associated DNA damage. $\mathrm{Mol}$ Cell Biol 31: 4379-4389.

GARNER, E. and COSTANZO, V. (2009). Studying the DNA damage response using in vitro model systems. DNA Repair (Amst) 8: 1025-1037.

HARLAND, R.M. and LASKEY, R.A. (1980). Regulated replication of DNAmicroinjected into eggs of Xenopus laevis. Cell 21: 761-771.

HASHIMOTO, Y., CHAUDHURI, A.R., LOPES, M. and COSTANZO, V. Rad51 protects nascent DNA from Mre11-dependent degradation and promotes continuous DNA synthesis. Nat Struct Mol Biol 17: 1305-1311.

HASHIMOTO, Y. and COSTANZO, V. Studying DNA replication fork stability in Xenopus egg extract. Methods Mol Biol 745: 437-445.

HASHIMOTO, Y. and COSTANZO, V. (2011). Studying DNA replication fork stability in Xenopus egg extract. Methods Mol Biol 745: 437-445.

HASHIMOTO, Y., PUDDU, F. and COSTANZO, V. RAD51- and MRE11-dependent reassembly of uncoupled CMG helicase complex at collapsed replication forks. Nat Struct Mol Biol 19: 17-24.

HASHIMOTO, Y., RAY CHAUDHURI, A., LOPES, M. and COSTANZO, V. (2010) Rad51 protects nascent DNA from Mre11-dependent degradation and promotes continuous DNA synthesis. Nat Struct Mol Biol 17: 1305-1311. 
JASIN, M. (2002). Homologous repair of DNA damage and tumorigenesis: the BRCA connection. Oncogene 21: 8981-8993.

JENSEN, R.B., CARREIRA, A. and KOWALCZYKOWSKI, S.C. Purified human BRCA2 stimulates RAD51-mediated recombination. Nature 467: 678-683.

LANA, E., MEGARBANE, A., TOURRIERE, H., SARDA, P., LEFRANC, G., CLAUSTRES, M. and DE SARIO, A. DNA replication is altered in Immunodeficiency Centromeric instability Facial anomalies (ICF) cells carrying DNMT3B mutations. Eur J Hum Genet 20: 1044-1050.

LAZZARO, F., NOVARINA, D., AMARA, F., WATT, D.L., STONE, J.E., COSTANZO, V., BURGERS, P.M., KUNKEL, T.A., PLEVANI, P. and MUZI-FALCONI, M. RNase $\mathrm{H}$ and postreplication repair protect cells from ribonucleotides incorporated in DNA. Mol Cell 45: 99-110.

LIU, Y. and WEST, S.C. (2002). Distinct functions of BRCA1 and BRCA2 in doublestrand break repair. Breast Cancer Res. 4: 9-13.

LOPES, M. (2009). Electron microscopy methods for studying in vivo DNA replication intermediates. Methods Mol Biol 521: 605-631.

LUKUSA, T. and FRYNS, J.P. (2008). Human chromosome fragility. Biochim Biophys Acta 1779: 3-16.

MECHALI, M. and KEARSEY, S. (1984). Lack of specific sequence requirement for DNA replication in Xenopus eggs compared with high sequence specificity in yeast. Cell 38: 55-64.

MIRKIN, E.V. and MIRKIN, S.M. (2007). Replication fork stalling at natural impediments. Microbiol. Molec. Biol. Rev. 71: 13-35.

OHTA, T., SATO, K. and WU, W. The BRCA1 ubiquitin ligase and homologous recombination repair. FEBS Lett 585: 2836-2844.

OZERI-GALAI, E., BESTER, A.C. and KEREM, B. The complex basis underlying common fragile site instability in cancer. Trends Genet 28: 295-302.

PAGEAU, G.J. and LAWRENCE, J.B. (2006). BRCA1 foci in normal S-phase nuclei are linked to interphase centromeres and replication of pericentric heterochromatin. J. Cell Biol. 175: 693-701.

RAY CHAUDHURI, A., HASHIMOTO, Y., HERRADOR, R., NEELSEN, K.J., FACHINETTI, D., BERMEJO, R., COCITO, A., COSTANZO, V. and LOPES, M. Topoisomerase I poisoning results in PARP-mediated replication fork reversal. Nat Struct Mol Biol 19: 417-423.
SANCHEZ, J.A., MAREK, D. and WANGH, L.J. (1992). The efficiency and timing of plasmid DNA replication in Xenopus eggs: correlations to the extent of prior chromatin assembly. J. Cell Sci. 103 (Pt 4): 907-918.

SCHALBETTER, S.A., MANSOUBI, S., CHAMBERS, A.L., DOWNS, J.A. and BAXTER, J. (2015). Fork rotation and DNA precatenation are restricted during DNA replication to prevent chromosomal instability. Proc Natl Acad Sci USA 112: E4565-E4570.

SCHLACHER, K., CHRIST, N., SIAUD, N., EGASHIRA, A., WU, H. and JASIN, M. Double-strand break repair-independent role for BRCA2 in blocking stalled replication fork degradation by MRE11. Cell 145: 529-542.

SHECHTER, D., COSTANZO, V. and GAUTIER, J. (2004). ATR and ATM regulate the timing of DNA replication origin firing. Nat Cell Biol 6: 648-655.

SUWAKI, N., KLARE, K. and TARSOUNAS, M. RAD51 paralogs: roles in DNA damage signalling, recombinational repair and tumorigenesis. Semin Cell Dev Biol 22: 898-905.

THORSLUND, T., MCILWRAITH, M.J., COMPTON, S.A., LEKOMTSEV, S., PETRONCZKI, M., GRIFFITH, J.D. and WEST, S.C. The breast cancer tumor suppressor BRCA2 promotes the specific targeting of RAD51 to single-stranded DNA. Nat Struct Mol Biol 17: 1263-1265.

THORSLUND, T. and WEST, S.C. (2007). BRCA2: a universal recombinase regulator. Oncogene 26: 7720-7730.

TOKMAKOV, A.A., TERAZAWA, Y., IKEDA, M., SHIROUZU, M. and YOKOYAMA, S. (2006). Coupled transcription-and-translation in Xenopusoocyte and egg extracts. J Biotechnol 125: 557-564.

TRENZ, K., ERRICO, A. and COSTANZO, V. (2008). PIX1 is required for chromosomal DNA replication under stressful conditions. EMBO J 27: 876-885.

TRENZ, K., SMITH, E., SMITH, S. and COSTANZO, V. (2006). ATM and ATR promote Mre11 dependent restart of collapsed replication forks and prevent accumulation of DNA breaks. EMBO J 25: 1764-1774.

WEST, S.C. (2003). Molecular views of recombination proteins and their control. Nat Rev Mol Cell Biol 4: 435-445.

WOODWARD, A.M., GOHLER, T., LUCIANI, M.G., OEHLMANN, M., GE, X., GARTNER, A., JACKSON, D.A. and BLOW, J.J. (2006). Excess Mcm2-7 license dormant origins of replication that can be used under conditions of replicative stress. $J$ Cell Biol 173: 673-683. 


\section{Further Related Reading, published previously in the Int. J. Dev. Biol.}

Control of timing of embryonic M-phase entry and exit is differentially sensitive to CDK1 and PP2A balance

Mohammed El Dika, Damian Dudka, Claude Prigent, Jean-Pierre Tassan, Malgorzata Kloc and Jacek Z. Kubiak

Int. J. Dev. Biol. (2014) 58: 767-774

Nuclear reprogramming in zygotes

Chanchao Lorthongpanich, Davor Solter and Chin Yan Lim

Int. J. Dev. Biol. (2010) 54: 1631-1640

Faithful reprogramming to pluripotency in mammals - what does nuclear transfer teach us?

Julien Maruotti, Alice Jouneau and Jean-Paul Renard

Int. J. Dev. Biol. (2010) 54: 1609-1621

Cyclin B2/cyclin-dependent kinase1 dissociation precedes CDK1 Thr-161 dephosphorylation upon M-phase promoting factor inactivation in Xenopus laevis cell-free extract Franck Chesnel, Franck Bazile, Aude Pascal and Jacek Z. Kubiak Int. J. Dev. Biol. (2007) 51: 297-305

Remodeling of sperm chromatin induced in egg extracts of amphibians.

C Katagiri and K Ohsumi

Int. J. Dev. Biol. (1994) 38: 209-216
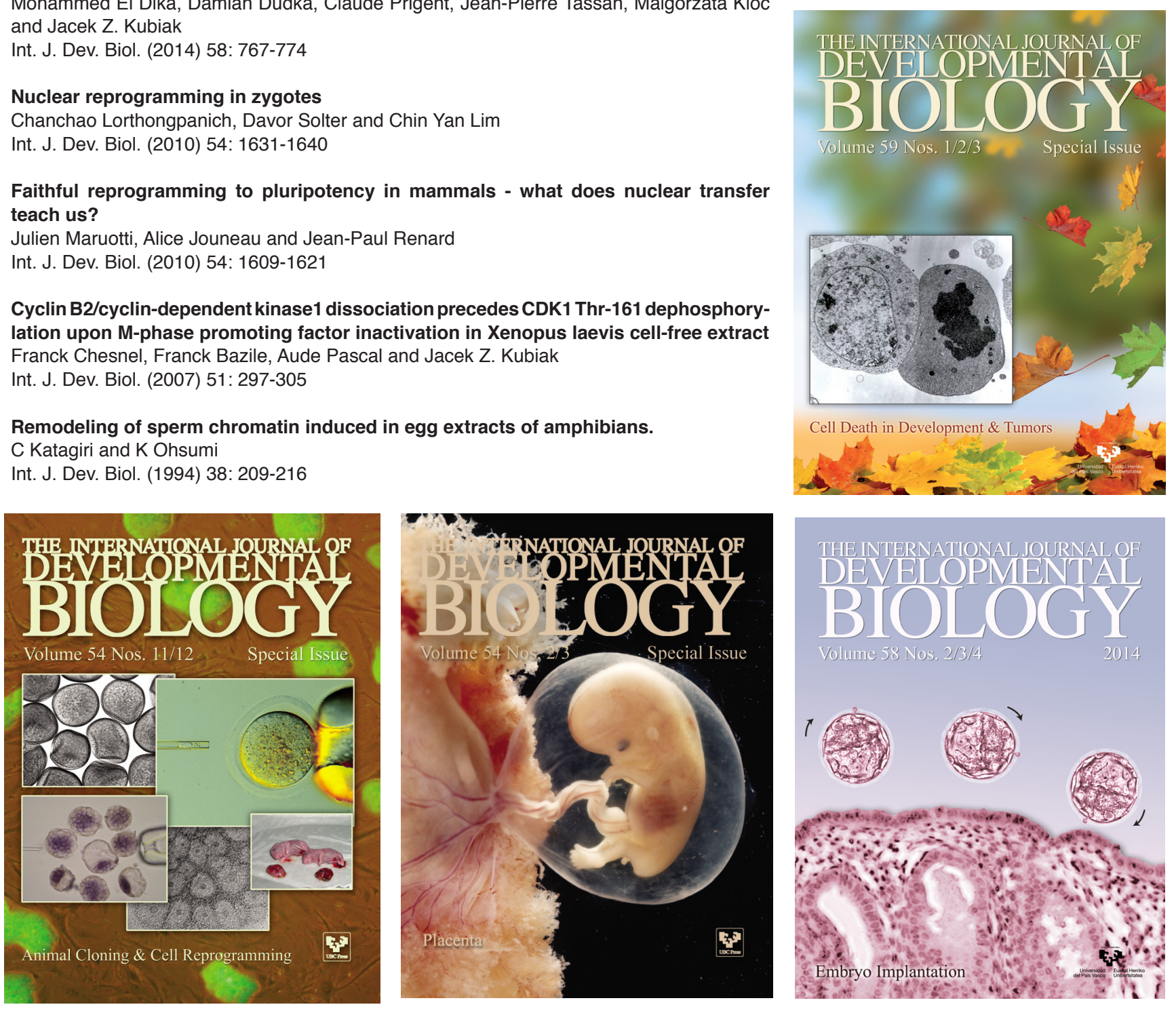